\title{
Lower radiation dose coronary CT angiography with new imaging technologies
}

\author{
Frank J. Rybicki
}

Received: 14 February 2009/Accepted: 19 February 2009/Published online: 6 March 2009

(C) Springer Science+Business Media, B.V. 2009

Developments in multidetector computed tomography (MDCT) are largely driven by the goal of high quality, reproducible, and accurate coronary CT angiography that is simple to perform and simple to interpret. Until recently, 64-slice CT was considered state-of-the-art; the 64 slices per gantry rotation were achieved with either 64-detector rows, or 32-detector rows and a strategy to double the slice number by alternating the focal spot of the X-ray source. These "64-generation" scanners offered considerable advantages over earlier technology such as superior spatial resolution and volume coverage. Temporal resolution was dramatically improved with the introduction of dual source CT.

All four CT vendors have released new products for cardiac CT since November 2007 at the annual meeting of the Radiological Society of North America (RSNA). Many developments have focused on lowering the patient radiation dose and applications for lower dose imaging. Some of these features were available for "64-generation" scanners, although doses using these technologies varied [1]. The group of new technologies can be called the "post-64" era [2]

\section{F. J. Rybicki ( $\square)$}

Applied Imaging Science Laboratory, Department of Radiology, Noninvasive Cardiovascular Imaging Program, Brigham and Women's Hospital \& Harvard Medical School, 75 Francis Street, Boston, MA 02115, USA

e-mail: frybicki@partners.org of coronary CT angiography (CTA). This collection of advances is difficult to name more descriptively because, unlike the evolution from 4 to 64 detector row coronary CTA, current CT releases are far from uniform, reflecting different approaches to image acquisition in the post-64 era. No single CT scanner offers the full portfolio of newest features. However, each offers coronary CTA with radiation doses reported (or marketed, for those new technologies that do not have peer-review publications to date) as comparable to, or lower than, conventional coronary angiography.

This Special Edition compiles peer-review low dose imaging articles from authors who use all major CT platforms. Below is a brief summary of new cardiac CT technologies as of February 2009. The descriptions are far from complete, but additional detail will be found in the pages to follow, including an overview of dose reduction techniques from Dr. Halliburton. Most cardiovascular imagers primarily use only one CT platform. This potentially introduces bias. Under normal circumstances, such bias could be considered detrimental. However, in this Special Issue the editorial staff has purposely solicited input from users of each vendor.

The most recent strategy (US approval in October 2008) for cardiac CT imaging features a scanner with two $4 \mathrm{~cm}$ detectors (dual source), each with 64 detector rows (128 slices each with an alternating focal spot) and a $285 \mathrm{~ms}$ gantry rotation time (Somatom Definition Flash, Siemens Medical Systems, 
Erlangen, Germany). Although this technology is very new and there is no peer-review literature to date, the vendor proposes the following acquisition characteristics. Imaging will use both X-ray tubes, a table speed of $43 \mathrm{~cm}$ per second, temporal resolution of $75 \mathrm{~ms}$, and a pitch value of 3.2. This is the maximum pitch that enables data acquisition without a spatial gap in the heart. The proposed lower dose imaging centered in time over a single period of diastole is a result of the fast acquisition. However, inherent to this technique is the fact that each slice will be obtained at a different phase of the cardiac cycle; for a $14 \mathrm{~cm}$ craniocaudal field of view, proposed imaging will span approximately $325 \mathrm{~ms}$.

In addition to the comments above, this scanner is briefly mentioned by Drs. Luaces and Blankstein whose articles focus on 64-slice dual source CT (Siemens Definition). Dr. Luaces and colleagues from the University of Pennsylvania focus on the role of optimizing $\mathrm{kVp}$ for individual patients' body habitus, as many patients can effectively be scanned with 100 as opposed to $120 \mathrm{kVp}$. Dr. Blankstein and colleagues use the same scanner in a two-acquistion protocol (stress followed by rest) to obtain myocardial perfusion as well as morphologic coronary imaging. Dr. Hein and colleagues demonstrate lower doses with prospective ECG gating as opposed to retrospective gating with dual source CT.

In late 2007 the GE Discovery 750HD received FDA approval. As with the new Siemens system, to date there are no images in the peer-review literature. As discussed by Dr. Budoff in this issue, the scanner proposes improved spatial resolution using new detector technology and iterative reconstruction. Dose reduction uses "step and shoot" prospective ECG gating introduced on earlier GE platforms. Dr. Earls shares his experience in over 2,000 cases of prospective ECG-gating, and Dr. Knowles and colleagues from the University of Michigan describe prospective gating in emergency room patients.

At the 2007 annual meeting of the RSNA, Philips Healthcare (Cleveland, OH, USA) announced US approval of the Brilliance iCT, featuring a $0.27 \mathrm{~s}$ rotation, $120 \mathrm{~kW} \mathrm{X}$-ray tube and generator, and a single $80.0 \mathrm{~mm}$ detector array $(128 \times 0.625 \mathrm{~mm})$. An alternating focal spot allows 256 slices from 128 detector rows. Cardiac applications for this system are described by Dr. Weigold and colleagues. Future studies will focus on validating the accuracy of coronary images for patients with heart rates greater than 70 beats per minute and variability greater than 10 beats per minute. As described by Mr. Walker of Philips Healthcare and colleagues from the Cleveland Clinic, the Brilliance iCT also features technology to reduce dose associated with wide area detectors for both prospectively and retrospectively ECG-gated cardiac acquisitions. This Special Issue also includes 256-slice applications for ECG-gated images of the chest, in particular the evaluation of aortic pathology, in an article by Dr. Hameed and colleagues from Indiana University.

In November 2007, also at the annual meeting of the RSNA, Toshiba Medical Systems (Tochigi-ken, Japan) introduced the AquilionONE Dynamic Volume CT with 320-detector rows and whole heart coverage. This technology does have prior reports in the peer-review literature [3] featuring single heart beat cardiac imaging, prospective ECG gating to lower dose ("step and shoot" is not required because of whole-heart coverage), and preliminary data on optimizing the phase window for prospective gating [4]. Dr. Kitagawa and colleagues from Johns Hopkins Division of Cardiology discuss perfusion imaging using 320-detector row CT, and an article from our group introduces single heart beat coronary shear stress mapping using a method to perform these computations on the entire coronary tree. Finally, Dr. Schuijf and colleagues from Leiden University discuss novel clinical applications in cardiac CT.

In summary, dose reduction in cardiac $\mathrm{CT}$ is timely and complex. Part of the complexity is an outgrowth of the different vendor platforms and dose reduction strategies. This special edition provides a broad-based collection of new peer-review articles to shed new insight on methods for lower-dose cardiac CT and new applications enabled by routine lower dose imaging.

\section{References}

1. Hausleiter J, Meyer T, Hermann F, Hadamitzky M, Krebs M, Gerber TC, McCollough C, Martinoff S, Kastrati A, Schomig A, Achenbach S (2009) Estimated radiation dose associated with cardiac CT angiography. JAMA 301(5):500-507. doi:10.1001/jama.2009.54

2. Otero HJ, Steigner ML, Rybicki FJ (2009) The "Post-64" era of coronary CT angiography: understanding new technology from physical principles. Radiol Clin North Am 47(1):79-90. doi:10.1016/j.rcl.2008.11.001 
3. Rybicki FJ, Otero HJ, Steigner ML, Vorobiof G, Nallamshetty L, Mitsouras D, Ersoy H, Mather RT, Judy PF, Cai T, Coyner K, Schultz K, Whitmore AG, Di Carli MF (2008) Initial evaluation of coronary images from 320-detector row computed tomography. Int J Cardiovasc Imaging 24(5):535546. doi:10.1007/s10554-008-9308-2
4. Steigner ML, Otero HJ, Cai T, Mitsouras D, Nallamshetty L, Whitmore AG, Ersoy H, Levit NA, Di Carli MF, Rybicki FJ (2009) Narrowing the phase window width in prospectively ECG-gated single heart beat 320-detector row coronary CT angiography. Int J Cardiovasc Imaging 25(1):85-90. doi: 10.1007/s10554-008-9347-8 